\title{
Literatura y activismo intelectual en la Argentina de los 80. Notas a partir de Lecturas críticas. Revista de Investigación y Teorías Literarias
}

\begin{abstract}
Resumen
A partir de Lecturas críticas. Revista de Investigación y Teorías Literarias, este trabajo describe diferentes formas del activismo intelectual desarrollado en Argentina en torno a la literatura, la teoría y la crítica literarias durante la última dictadura militar. Los "grupos de estudio" (o la llamada "universidad de las catacumbas”), además de hacer circular y discutir literatura y teorías prohibidas, producen conferencias, revistas así como un colectivo crítico que, con el retorno democrático, renovará la enseñanza y la investigación universitarias en una zona importante del país. Pero por otro lado, durante la dictadura, estas formaciones y las derivas que generan en el campo actúan como núcleos de resistencia desajustados de la moral y de las ideologías oficiales.
\end{abstract}

Palabras claves Literatura, universidad de las catacumbas, Argentina, dictadura, teoría y crítica literarias

\begin{abstract}
Taking Lecturas críticas. Revista de Investigación y Teorías Literarias as a starting point, this article describes different forms of intellectual engagement in relation to literature, literary theory and criticism in Argentina during the 19761983 military dictatorship. "Study groups" -or the so-called "university of the catacombs"--, apart from circulating and discussing censored literature and
\end{abstract}


theories, were responsible for the organization of lectures, the publication of journals and the creation of a critical profession which, on the restoration of democracy, renewed university teaching and research in a wide area of the country. But on the other hand, during the dictatorship, these formations and the scholarship they generate in the field constitute kernels of resistance, which are not aligned with official morals and ideology.

Key Words Literature, university of the catacombs, Argentina, dictatorship, literary theory and criticism

"El deseo nace del derrumbe” es la epigramática sentencia usada para rotular diferentes tipos de archivo de la “obra” de Roberto Jacobi: un libro (cf. Longoni), una muestra en el Museo Reina Sofía de Madrid durante al año 2011 que reúne parte de su producción. Pocas frases breves han logrado concentrar tanto: más allá del contexto que la rodea, la ocurrencia explica, entre otras cosas, lo que pudo hacerse en la Argentina desde el arte, su teoría y su crítica a pesar de las acciones devastadoras de las dos últimas dictaduras (la de Onganía, entre 1966 y 1973, y el llamado "Proceso de Reorganización Nacional”, entre 1976 y 1983) que, si bien con intervalos, siguieron durante las gestiones posteriores hasta derivar en el estallido social de 2001.

Este artículo se desprende de una investigación centrada en las operaciones de lectura desplegadas en las cátedras de Literatura argentina y de Teoría literaria en las carreras universitarias de letras durante la Argentina de la posdictadura, es decir, entre 1983 y $2003^{1}$. Un análisis que se trama en diálogo con los dilemas de los campos intelectual, literario y artístico (Bourdieu Choses, Las reglas) en el marco económico, social y cultural ${ }^{2}$. Punto de mira necesario si

${ }^{1}$ Para una justificación detallada de los criterios que permiten establecer el período de la posdictadura argentina entre 1983 y 2003, ver Gerbaudo.

2 Tomo el concepto “campo" de Pierre Bourdieu junto a las observaciones de Federico Neiburg y Mariano Ben Plotkin, Gisèle Sapiro (“Entretien”) y Joseph Jurt. Plotkin observa que Bourdieu "caracteriza a los campos como espacios de posiciones y combates por la posesión de un cierto 
lo que se pretende es armar una "vista del pasado” (Sarlo Tiempo) atenta a las intervenciones realizadas desde las aulas de la universidad pública en términos de reorganización del entramado sociocultural a partir de lo que se hace con esa forma del arte llamada "literatura” y con las teorías desde las que se enseña a leerla.

En este escrito me detengo en la lectura de una “formación” (Williams) que, junto a otras, ayuda a comprender lo que pudo y puede hacerse en la universidad argentina desde el retorno democrático, a pesar de las condiciones de producción que dominaron hasta 2003. Esta formación deriva de una tradición clandestina llamada "universidad de las catacumbas” o "grupos de estudio": espacios marginales que, por fuera de toda institución, generaron durante los años setenta los movimientos de apropiación y de reinvención teórica y crítica que explican el estallido teórico y literario que se observa en las instituciones en las que enseñaron sus participantes una vez restaurada la democracia.

Lecturas críticas. Revista de Investigación y Teorías Literarias es producto de una formación que porta huellas de los "maestros" que trabajaron en la "universidad de las catacumbas” o "grupos de estudio” durante la dictadura: Beatriz Sarlo, Josefina Ludmer, Nicolás Rosa, Ricardo Piglia, Eduardo Romano, Carlos Altamirano.

“Allí veíamos marcos teóricos que no se estudiaban en la universidad: Freud y La interpretación de los sueños, el formalismo ruso, sociología de la literatura, Benjamin, Gramsci y las culturas populares”, subraya Ana María

capital simbólico definido internamente” (14). Y agrega: "en contextos periféricos [...] el concepto de autonomía (central para la definición de los campos) se vuelve más borroso que en el caso de Francia, espacio social y cultural para el cual Bourdieu construyó su análisis” (14). A la luz de las discusiones actuales sobre la autonomía de la literatura y las leyes del campo literario (Ludmer, Aquí; Sapiro La responsabilité, “La responsabilité”, Bud, Boltanski), las crisis mundiales que afectaron en todas sus estructuras a los países que producen buena parte de las teorías que utilizamos, la discusión sobre ensambles teóricos sólo aparentemente incompatibles (cf. Pinto) y los conceptos “apropiación”, "herencia” (Derrida) y “uso” (Laugier) que promueven la creatividad teórica e investigativa atenta a los contextos de re-invención, realizo estos cruces disciplinares y teóricos, necesarios para desarrollar el problema planteado por la investigación (además de las hipótesis teórico-críticas, decido también correr este riesgo en el plano epistemológico). 
Zubieta mientras reconstruye sus años de estudio con Ludmer. Años de los que Ana María Camblong destaca su intensidad teórico-política tanto como su carácter clandestino: “a las viviendas particulares en las que nos reuníamos llegábamos de a uno, con horarios un poco diferentes, y nos íbamos del mismo modo". "En tiempos de la dictadura la universidad nos daba lo peor”, recalca Graciela Montaldo. Su relato pone en evidencia la densidad del momento. Se trabajaba “de cualquier cosa” mientras paralelamente se asistía a la universidad y se participaba activamente de los grupos de estudio: “con Sarlo leíamos a Bajtin, a los formalistas en italiano, Marxismo y literatura de Williams sin sus tapas para poder fotocopiarlo y hacerlo circular sin mayores inconvenientes”. Y agrega: "Leíamos mal las lenguas extranjeras pero predominaba una pasión por descifrar eso que no conocíamos”.

Provenientes de distintos “grupos de estudio”, en 1980, Nora Domínguez, Alan Pauls, Silvia Prati, Renata Rocco-Cuzzi, Adriana Rodríguez Pérsico, Alfredo Rubione y Mónica Tamborenea sacan a la calle Lecturas críticas. Una revista con dos números: el segundo, de 1984. A pesar de su carácter efímero, la intervención es importante por motivos diversos.

En primer lugar, por la valentía con que enfrentan el estado de las cosas haciendo otras para revertirlo. En una suerte de editorial, en la primera página del primer número, presentan la revista como "el paso a la escritura de varios años de trabajo conjunto” (“paso a la clandestinidad”, “paso a la lucha armada”, “paso a la escritura”: pongo en serie diferentes formas de acción de la época, enlazadas por un cambio de rumbo, por un viraje). Y agregan: “Este paso quería dejar atrás el aislamiento: uno de los peligros más concretos que amenazan a los grupos dedicados al debate de cuestiones vinculadas con la cultura” (3). Imaginemos la escena: estamos en 1980, lejos aún del golpe final que el fracaso de la guerra por Malvinas significó para la dictadura. Quienes escriben se reconocen como un grupo de investigación dedicado al problema del realismo que decide cambiar de soporte: de la "discusión oral a la escritura” (3). Y lo hacen con consciencia del riesgo que corren: "Es sabido que, en medios como el nuestro, sometidos a tantos 
vaivenes históricos, el acceso a la escritura resulta problemático y decisivo. A las determinaciones externas se agregan, además, los riesgos individuales que supone la práctica de escribir” (3).

Repasar algunos datos ayudará a dimensionar el peligro así como el necesario trabajo que vendrá luego para reponer algo de tamaña devastación. Una destrucción que comprende desde obras puntuales a políticas editoriales, de traducción, de divulgación y de mercado ya que el circuito represivo incluía no sólo a escritores, periodistas, profesores o críticos sino también a editores, libreros y traductores. Por ejemplo, el 2 de mayo de 1979, es decir, un año antes de que se publicara el primer número de Lecturas críticas, la Dirección de Publicaciones elaboró un listado destinado a la Dirección General de Provincias del Ministerio del Interior con los títulos cuyo ingreso al país estaba prohibido. El punteo pretendía, además, identificar sus lugares de circulación en el interior y retirarlos. Los criterios de clasificación del material, elaborados por la Secretaría de Inteligencia del Estado (SIDE), eran: "FÓRMULA 1: Carece de referencias ideológicas contrarias a los principios sustentados por nuestra Constitución Nacional"; "FÓRMULA 2: Contiene referencias ideológicas que atentan contra los principios sustentados por nuestra Constitución Nacional”; "FÓRMULA 3: Propicia la difusión de ideologías, doctrinas o sistemas políticos, económicos o sociales tendientes a derogar los principios sustentados por nuestra Constitución Nacional” (cf. Invernizzi y Gociol 69). Por ejemplo, en la Fórmula 2 se incluyeron Introducción al pensamiento científico de Gastón Bachelard, De Sarmiento a Cortázar de David Viñas, Estructuralismo y psicoanálisis (una edición de José Sazbón con artículos de Louis Althusser, entre otros). Entre las editoriales clasificadas como "marxistas y/o con más de 50\% de obras marxistas" estaban, entre otras, Corregidor, Crisis, De la Flor, Centro Editor de América Latina, Fondo de Cultura Económica, Jorge Álvarez, Nueva Visión, Paidós, La Rosa Blindada, Siglo XXI, Seix Barral, Universitaria Córdoba, Universitaria de Mar del Plata (73). Entre los títulos prohibidos es sintomática la censura de "Una torre de cubos” de Laura Devetach por su "ilimitada fantasía” y reveladoras del 
alcance de la persecución las observaciones sobre un libro para enseñar francés como lengua extranjera. Puntualmente lo que inquieta son referencias al Mayo del 68: "'retratos en los muros de Lenin, Mao y el Che Guevara y ramos de flores rojas en las estatuas de Víctor Hugo y Pasteur’” (120).

Este repaso tiene sentido a la luz de la revisión de los temas, autores y preguntas introducidos en 1980 por Lecturas críticas. Otro punto que pone en valor la intervención. Hacer circular la bibliografía teórica o el estado de la cuestión sobre los temas nodales de cada número (la parodia, en el primero; los “géneros menores”, en el segundo), presentar reseñas de revistas específicas, de textos teóricos o literarios y entrevistar a los escritores y críticos que accionan el campo intelectual argentino y latinoamericano son operaciones especialmente apreciables dado que se realizan en un tiempo previo a Internet, es decir, con otros canales y circuitos, no sólo de comunicación sino de acceso (liso y llano) a la información. Valor acrecentado por la intensidad que le confieren las particulares circunstancias bajo las cuales se hacía circular el material. Dice la editorial: "Se encontrarán aquí ciertas líneas de investigación, y ciertos objetos de estudio (en especial, la literatura argentina), que en los últimos años no han podido recibir la atención que merecen” (3). Forma no tan velada de hacer alusión a las circunstancias opresivas bajo las cuales se realizaba, a pesar de todo, la producción (los testimonios y los datos citados más arriba son sólo muestras de un complejo estado de las cosas). La aclaración que sigue manifiesta la diversidad de perspectivas que confluyen en la revista (nota que, por su apertura multicultural, podría haber sido objeto de perspicacias): "Reconocemos como supuesto común que la crítica consiste en una actividad productiva que supone el manejo de materiales (elaboraciones teóricas, conceptos, doctrinas, códigos) de procedencia diversa, y que pertenecen tanto a nuestra tradición, como a otras lenguas y culturas” (3).

En este trabajo apunto las operaciones de importación teórica como de difusión de la literatura y la crítica argentina más riesgosas realizada por este grupo. Si bien me detengo exhaustivamente en el primer número (básicamente 
porque introducir el segundo me obligaría a precisar detalles del campo cultural y excedería la extensión admitida para esta presentación), me apuro a aclarar que el segundo número queda pendiente para un próximo trabajo ya que lo que allí se observa es un redoble de la apuesta, especialmente en su tono político (otra operación que merece un análisis minucioso: los avatares del país no daban demasiadas garantías a aquella flamante democracia en julio de 1984, fecha en la que ese segundo número sale a la calle).

Entre esas operaciones de riesgo aludidas en el párrafo anterior destaca el esclarecimiento teórico del concepto de "parodia” por Alan Pauls que introduce los desarrollos de Mijail Bajtin con algunas referencias al formalismo ruso y a Julia Kristeva. En uno de sus pasajes aclara que, a diferencia de los formalistas, Bajtin se interesa por "el proceso de construcción" de la novela ocupándose de "las condiciones específicas de posibilidad" (7). Apunte valiente en la Argentina de 1980. La correlación es inevitable: ¿cuáles fueron las condiciones de posibilidad de la novela en el país entre 1976 y 1980 con la clausura de editoriales, la censura y quema de libros, la persecución y el exterminio de editores, libreros, escritores, traductores, periodistas, profesores, críticos? Pauls subraya además que, por contraste con el formalismo que encontraba en la parodia un "juego de procedimientos”, Bajtin reconoce en ella "la manifestación concreta de un conflicto ideológico” (10).

Esta introducción de un autor materialista, retomando no sólo sus postulados sino sus circunstancias de producción, recorre la revista completa. Una insistencia peligrosa que conecta este artículo de apertura con la reseña de "Bakhtine et l'alterité", firmado por Tzvetan Todorov e incluido en el número 40 de la revista Poétique. Alfredo Rubione firma la reseña. Llama la atención que de todo ese número de Poétique sólo tome ese artículo. Se puede hipotetizar que esto obedece a un interés particular por hablar de aquello a lo que Todorov remite. Una cadena de envíos ya que Todorov cuenta los avances de un libro que publicará el año siguiente: en 1981 saldrá a la venta Mikhaïl Bakhtine. Le principe dialogique. Los énfasis de Rubione se saltean la escritura de Todorov para extraer 
lo que puede de lo que éste a la vez cita de Bajtin. Una operación que el propio Todorov realizará en su libro, eligiendo callar para dejarle la palabra a aquel que no tuvo otra opción más que el silencio. Circunstancia de producción que ambos, Rubione en la reseña y Todorov en el artículo, en su libro (Mikhaïl) y en su divulgación anticipada ("Bakhtine”), ligan directamente con las categorías centrales de la obra bajtiniana: el principio dialógico es la respuesta sintomática y paradojal que se deriva del encierro, de la imposibilidad del diálogo, de la ausencia de interlocución ${ }^{3}$. La diferencia es que, mientras Todorov pone a circular sus tesis en la Francia renovada por los aires de izquierda de François Mitterrand, Rubione pone a circular las suyas bajo la dictadura de Jorge Rafael Videla. Y no se ahorra adjetivos. Por ejemplo: “impresionante” es el que usa para expresar su conmoción ante la paradoja de que Bajtin, “el hombre para quien la ausencia de respuesta era el mal absoluto, el infierno, sufriera tal suerte: la de no haber recibido jamás respuesta” (69).

En la misma línea de riesgo se inscribe la decisión de publicar la entrevista a Ricardo Piglia cuya crítica a la tendencia a “desocializar” la lectura de la literatura bajo el amparo de las teorías de la autonomía y de cierta etapa del formalismo ruso se conjuga con su adscripción a las perspectivas de Walter Benjamin y de Bertolt Brecht en cuya obra encuentra “la primera gran síntesis” de una teoría materialista (39).

Las operaciones con y sobre la literatura no se quedan atrás. No sólo por lo que se lee sino por cómo se lee. Por ejemplo, en "El Fausto criollo: una doble

${ }^{3}$ Dice Todorov en la contratapa de Poétique, mientras anuncia el libro por-venir: "On pourrait accorder sans trop d’hésitations deux superlatifs à Mikhäil Bakhtine, en affirmant qu'il est le plus important penseur soviétique dans le domaine des sciences humaines, ainsi que le plus grand théoricien de la littérature au vingtième siècle. A une époque de surpublication fiévreuse, on peut admirer la détermination de Bakhtine, qui développe pendant cinquante ans les mêmes pensées, tout en les enfermant dans ses dossiers. Mais on peut se demander aussi dans quelle mesure toute la théorie du dialogue n'est pas née du désir de comprendre cet état insupportable -l'absence de réponse... C'est pour remédier à cette carence que j'ai essayé, dans ces pages, de faire entendre à nouveau la voix de Bakhtine : pour que le dialogue puisse enfin commencer ”. Cada una de estas afirmaciones se despliegan y se expanden en los dos primeros capítulos del libro que se publica el año siguiente (cf. "Introduction” y "Biographie”). 
mirada”, Nora Domínguez y Beatriz Masine analizan los procesos paródicos de Estanislao Del Campo en términos de clase y de jerarquía. Afirman: "Es la civilización la que da su propia versión de la barbarie” (25). Un enunciado que se disemina en cruce con el epígrafe de Jorge Luis Borges tomado de "Nota sobre (hacia) Bernard Shaw”: "Una literatura difiere de otra, ulterior o anterior, menos por el texto que por la manera de ser leída” (20). Un pasaje atento a los anteojos desde los cuales aprendemos a mirar y que le devuelve la fuerza configuradora a las preguntas sobre los objetos (entre ellos, los que ubicamos a cada lado de la división civilización / barbarie).

Un rápido repaso sobre qué se lee muestra la operación perseguida a través de la selección realizada: Alfredo Rubione escribe sobre “El niño proletario” de Osvaldo Lamborghini; Nora Domínguez reseña Nadie nada nunca de Juan José Saer; Silvia Prati, Mis muertos punk de Rodolfo Fogwill. Como otra forma del envío a sus obras se publican entrevistas a Nicolás Rosa, Osvaldo Lamborghini, Severo Sarduy y la ya citada a Ricardo Piglia. En el apartado "Ficción”, se incluye un inclasificable y desopilante texto de Alberto Laiseca: "Por favor, ¡plágienme!”.

Algunas otras zonas de riesgo: en las entrevistas se habla de Sade, de Luis Gusmán (algo más que autores incómodos para el discurso oficial de la época). En su artículo sobre "El niño proletario”, Rubione subraya el carácter "escandalosamente amoral e insensible” de un narrador "literalmente perverso" (28). En sus notas sobre Nadie nada nunca, ese texto oblicuo sobre el terrorismo de Estado en su versión santafesina, Nora Domínguez escribe una oración peligrosamente ambivalente que confronta esa narración de la "nada” con "la violencia”; la mansedumbre del río contra las matanzas de caballos y la del Caballo, seudónimo del jefe de policía del lugar. Si bien atenuadas por las frases que siguen, en un pasaje define a la violencia como "punto de juntura entre la literatura y la historia” (64). Se podría pensar en la historia narrada; se podría pensar en la historia, a secas. 
Una última operación: la inscripción del deseo de polémicas. El hilván que lleva de Bajtin y la referencia a sus circunstancias de escritura se sigue hasta la frase que cierra la minuciosa descripción que Adriana Rodríguez Pérsico realiza de un homenaje a Jaime Rest organizado por el grupo nucleado en torno a la revista Punto de vista bajo la forma de conferencias de Josefina Ludmer, Beatriz Sarlo, Ricardo Piglia y Eduardo Romano (otra vez, los “maestros”). Más allá de los temas que se llevan a la escena pública, es la posibilidad de confrontar en ese espacio lo que parece añorarse: “Nos queda desear y trabajar para que propuestas como ésta se tornen realidad y no asombren” ("Homenaje” 62). Los significantes dicen más que mil parafraseos: “desear” y “trabajar” para que hechos como estos “no asombren”. “Nos queda”: legado y mandato.

Actuando esa máxima (la de “trabajar” para que una conferencia pública no sea un evento de excepción), aparece esta revista que, si bien Adriana Rodríguez Pérsico describe como una “experiencia de jóvenes” que "no dependió de ninguno de nuestros maestros” (cf.”Cuestionario”) (cuestión que es importante subrayar dado el arrojo de hacer circular públicamente materiales de este calibre en los 80), es deudora de las operaciones practicadas en el círculo clandestino de la "universidad de las catacumbas". Hay tres puntos donde esto se deja entrever: en el canon literario, en el canon teórico y en el tipo de intervención crítica. Es decir: se lee básicamente una literatura de vanguardia, se está atento al estado de la cuestión de la teoría literaria haciendo un esfuerzo por incluirse en un debate que traspasa las fronteras de Argentina (una marca de la impronta del Instituto Di Tella ${ }^{4}$ ) y se busca la interlocución crítica con sello propio, es decir, con sello polémico. Valga como síntesis de esa actitud dos reseñas: la de Renata RoccoCuzzi, que realiza una implacable crítica al número 7 de la revista Espiral (edición monográfica sobre humor, ironía y parodia) y la de Alan Pauls que, con

4 «L'expression 'el Di Tella' est en Argentine [...] synonyme d'un nouveau monde social et culturelle dont la valeur repose sur la 'production nationale de niveau internationale' en sciences et en arts » (Neiburg y Plotkin 372). Ver, sobre esta misma cuestión, Longoni y Mestman, Rizzo. 
elegancia pero sin sutilezas, se aparta de la conciliadora actitud crítica de Roland Barthes en el Coloquio de Cerisy-la Salle de junio de 1977 (cf. Compagnon).

Del segundo número sólo agrego que sus contenidos están en relación con las intervenciones que los integrantes del grupo realizarán en diferentes equipos de cátedra durante la democracia en la Universidad de Buenos Aires: algunos junto a Enrique Pezzoni que inaugurará una nueva forma de leer la Teoría Literaria desde América Latina en “Introducción a la Literatura” (una apropiación que destierra, como bien lo señala Jorge Panesi, la idea de la “caja de herramientas” o la pretensión hermenéutica para poner en valor la potencia diseminatoria de la literatura que introduce a partir de las teorías de Bajtin, Jacques Derrida, Paul De Man); otros, junto a Josefina Ludmer cuyas enseñanzas desde su concurrido Seminario “Algunos problemas de Teoría Literaria” pretenden formar lectores autónomos, al margen de los vaivenes tanto de la academia como del mercado (Modos de ver de John Berger signa la epistemología literaria que Ludmer se inventa hacia 1985 posicionando a la crítica como una forma de “activismo cultural”); otros, junto a Beatriz Sarlo, cuya atención estará puesta en las vanguardias teóricas (Pierre Bourdieu, Raymond Williams y Edward Said) y literarias (básicamente, Saer como "bandera” [cf. "Entrevista”] junto a Osvaldo Lamborghini, César Aira, Rodolfo Fogwill, Rodolfo Walsh, Julio Cortázar, Héctor Tizón), en su difusión a partir de sus clases, libros, traducciones y artículos en revistas y periódicos.

En síntesis, no puede explicarse la explosión teórica y literaria que vive la Universidad de Buenos Aires durante los primeros años de la democracia (es decir, durante el "primer momento" de la posdictadura, entre 1984 y 1986 [cf. Gerbaudo]) sin este trabajo previo que genera, desde la clandestinidad, las condiciones para ese florecimiento. Un trabajo en núcleos de resistencia que, sintomáticamente, son llamados en esta revista "grupos parauniversitarios”5: una

\footnotetext{
${ }^{5}$ Esta es la expresión que emplea el grupo en una breve referencia biográfica, al inicio de la entrevista a Josefina Ludmer publicada en el segundo número (cf. Ludmer “Un género” 46).
} 
CATEDRAL Tomada: Revista de crítica literaria latinoamericana / Journal of Latin American Literary Criticism Literatura y activismo intelectual en la Argentina de los 80. Notas a partir de Lecturas críticas. Revista de Investigación y Teorías Literarias

suerte de contraofensiva a las acciones de los "grupos paramilitares" del terrorismo estatal. Una contraofensiva cultural, con la palabra como arma.

\section{Bibliografía}

Boltanski, Luc. "Les conditions d'apparition du roman policier ». Journées Internationales d'Étude 'Autonomie de la littérature et ethos démocratique. 12-13 décembre. Observatoire du Livre et de l'écrit : Paris, 2011.

Bourdieu, Pierre. Choses dites. Paris: Minuit, 1987.

---. Las reglas del arte. Génesis y estructura del campo literario. Barcelona: Anagrama, 1995.

Bud, Crina. "La démocratie populaire et l'esthétisme irresponsable. L’histoire roumaine d'une faute littéraire ». Journées Internationales d'Étude 'Autonomie de la littérature et ethos démocratique. 12-13 décembre. Observatoire du Livre et de l'écrit : Paris, 2011.

Compagnon, Antoine (dir.). Prétexte: Roland Barthes. Paris: Centre Culturel International de Cersisy-La-Salle, 1978.

Derrida, Jacques. “A corazón abierto”. En ¡Palabra! Instantáneas filosóficas. Trotta, Madrid, 2001. 13-48.

Domínguez, Nora y Beatriz Masine. "El Fausto criollo: una doble mirada”. Lecturas críticas. Revista de Investigación y Teorías Literarias 1 (1980): 20-26.

Domínguez, Nora y otros. (Editorial). Lecturas críticas. Revista de Investigación y Teorías Literarias 1 (1980): 3.

Gerbaudo, Analía. "La literatura en la universidad argentina (1984-1986). Intervenciones desde una política de la exhumación”. Moderna språk 105 (2011): 91-106.

Invernizzi, Hernán y Judith Gociol. Un golpe a los libros. Represión a la cultura durante la última dictadura militar. Buenos Aires: Eudeba, 2002.

Jurt, Joseph. "L’apport de la théorie du champ aux études littéraires », en Pierre Bourdieu, sociologue. Paris : Fayard, 2004. 255-277.

Lamborghini, Osvaldo. "El lugar del artista” (Entrevista). Lecturas críticas. Revista de Investigación y Teorías Literarias 1 (1980): 48-52.

Laugier, Sandra. Wittgenstein. Les sens de l'usage. Paris. Vrin, 2009.

Longoni, Ana. (editora) El deseo nace del derrumbe. Roberto Jacobi: acciones, conceptos, escritos. Buenos Aires-Madrid: La Central-Adriana HidalgoMuseo Reina Sofía, 2011.

Longoni, Ana y Mariano Mestman. Del Di Tella a 'Tucumán arde’. Vanguardia artística y política en el '68 argentino. Buenos Aires: Eudeba, 2000. 
Ludmer, Josefina. "Un género es siempre un debate social” (Entrevista). Lecturas críticas. Revista de Investigación y Teorías Literarias 2 (1984): 46-51.

---. Aquí América Latina. Una especulación, Buenos Aires: Eterna Cadencia, 2010.

Neiburg, Federico y Plotkin, Mariano. «L’importation de la science économique américaine en Argentine ». Pour une histoire des sciences sociales. Hommage à Pierre Bourdieu. Johan Heilbron, Remi Lenoir, Gisèle Sapiro, directores. Paris: Fayard, 2004. 367-385.

Pauls, Alan. “Tres aproximaciones al concepto de parodia”. Lecturas críticas. Revista de Investigación y Teorías Literarias 1 (1980): 7- 14.

Piglia, Ricardo. "Parodia y propiedad” (Entrevista). Lecturas críticas. Revista de Investigación y Teorías Literarias 1 (1980): 37-40.

Pinto, Louis. "Volontés de savoir. Bourdieu, Derrida, Foucault». Pour une histoire des sciences sociales. Hommage à Pierre Bourdieu. Paris : Fayard, 2004. 19-48.

Plotkin, Mariano Ben. La privatización de la educación superior y las ciencias sociales en Argentina. Un estudio de las carreras de Psicología y Economía. Buenos Aires: CLACSO, 2006.

Rizzo, Patricia. Instituto Di Tella. Experiencias '68. Buenos Aires: Proa, 1998.

Rodríguez Pérsico, Adriana. “Homenaje a Jaime Rest”. Lecturas críticas. Revista de Investigación y Teorías Literarias 1 (1980): 60-62.

Rosa, Nicolás. "El Otro textual” (Entrevista). Lecturas críticas. Revista de Investigación y Teorías Literarias 1 (1980): 41-45.

Rubione, Alfredo. "Lo paródico en 'El niño proletario'”. Lecturas críticas. Revista de Investigación y Teorías Literarias 1 (1980): 27-31.

Sapiro, Gisèle. «Entretien de Pierre Bourdieu avec Gisèle Sapiro », en Pierre Bourdieu, sociologue. Paris : Fayard, 2000. 79-91.

---. La responsabilité de l'écrivain. Littérature, droit et morale en France (XIXeXXe siècle). Paris : Seuil, 2011.

---. « La responsabilité de l'écrivain doit-elle limiter ses droits ? Les étapes de la reconnaissance sociale de l'autonomie de la littérature en France (XIXe$\mathrm{XXe}$ siècles) ». Journées Internationales d'Étude 'Autonomie de la littérature et ethos démocratique. 12-13 décembre. Observatoire du Livre et de l'écrit : Paris, 2011.

Sarduy, Severo. "Reírse de la risa” (Entrevista). Lecturas críticas. Revista de Investigación y Teorías Literarias 1 (1980): 46-47.

Sarlo, Beatriz. Tiempo pasado. Cultura de la memoria y giro subjetivo. Una discusión. Buenos Aires: S. XXI, 2005.

Todorov, Tzvetan. Contratapa. Poétique. Revue de théorie et d'analyse littéraires 46 (1981). 
---. Mikhaïl Bakhtine. Le principe dialogique suivi de Écrits su Cercle de Bakhtine. Paris : Du Seuil, 1981.

Williams, Raymond. Marxismo y literatura, Barcelona: Península, 1980.

Zizek, Slavoj. El acoso de las fantasías, México: S. XXI, 1999.

\section{Archivos}

Camblong, Ana María. “Entrevista personal”, 2009. Investigación CICCONICET. CD-ROM.

Domínguez, Nora y otros. Lecturas críticas. Revista de Investigación y Teorías Literarias 1 (1980).

---. Lecturas críticas. Revista de Investigación y Teorías Literarias 2 (1984).

Ludmer, Josefina. Clases del Seminario “Algunos problemas de Teoría Literaria”, 1985 (UBA). Investigación CIC-CONICET. CD-ROM.

---. Programa del Seminario “Algunos problemas de Teoría Literaria”, 1985 (UBA). Investigación CIC-CONICET. CD-ROM.

Montaldo, Graciela. “Entrevista personal”, 2010. Investigación CIC-CONICET. CD-ROM.

Pezzoni, Enrique. Programa de la cátedra "Introducción a la Literatura”, 1985 (UBA). Investigación CIC-CONICET. CD-ROM.

Rodríguez Pérsico, Adriana. “Cuestionario” (vía e-mail), 2012. Investigación CIC-CONICET. CD-ROM.

Sarlo, Beatriz. Programa de la cátedra “Literatura argentina II”, 1984 (UBA). Investigación CIC-CONICET. CD-ROM.

---. “Entrevista personal”, 2009. Investigación CIC-CONICET. CD-ROM.

Zubieta, Ana María. “Entrevista personal”, 2008. Investigación CIC-CONICET. CD-ROM. 